\title{
Article \\ Analysis of a Nonlinear Technique for Microwave Imaging of Targets Inside Conducting Cylinders
}

\author{
Alessandro Fedeli * ${ }^{D}$, Matteo Pastorino, Andrea Randazzo $\mathbb{D}$ and Gian Luigi Gragnani \\ Department of Electrical, Electronic, Telecommunications Engineering, and Naval Architecture, \\ University of Genoa, 16145 Genoa, Italy; matteo.pastorino@unige.it (M.P.); andrea.randazzo@unige.it (A.R.); \\ gianluigi.gragnani@unige.it (G.L.G.) \\ * Correspondence: alessandro.fedeli@unige.it
}

check for updates

Citation: Fedeli, A.; Pastorino, M; Randazzo, A.; Gragnani, G.L. Analysis of a Nonlinear Technique for Microwave Imaging of Targets Inside Conducting Cylinders. Electronics 2021, 10, 594. https://doi.org/ 10.3390/electronics10050594

Academic Editor: Reza K. Amineh

Received: 2 February 2021

Accepted: 27 February 2021

Published: 4 March 2021

Publisher's Note: MDPI stays neutral with regard to jurisdictional claims in published maps and institutional affiliations.

Copyright: (c) 2021 by the authors. Licensee MDPI, Basel, Switzerland. This article is an open access article distributed under the terms and conditions of the Creative Commons Attribution (CC BY) license (https:/ / creativecommons.org/licenses/by/ $4.0 /)$.
Abstract: Microwave imaging of targets enclosed in circular metallic cylinders represents an interesting scenario, whose applications range from biomedical diagnostics to nondestructive testing. In this paper, the theoretical bases of microwave tomographic imaging inside circular metallic pipes are reviewed and discussed. A nonlinear quantitative inversion technique in non-Hilbertian Lebesgue spaces is then applied to this kind of problem for the first time. The accuracy of the obtained dielectric reconstructions is assessed by numerical simulations in canonical cases, aimed at verifying the dependence of the result on the size of the conducting enclosure and comparing results with the conventional free space case. Numerical results show benefits in lossy environments, although the presence and the type of resonances should be carefully taken into account.

Keywords: inverse scattering; tomography; conducting cylinders

\section{Introduction}

Since the end of the past century [1], inverse electromagnetic scattering problems aimed at microwave imaging have experienced a widespread interest among researchers. Indeed, while still at a prototyping level in many applications, microwave imaging is evolving to a mature technique in many fields of civil engineering, industrial testing, medicine, and so on [2,3]. Although a comprehensive review about the topic is outside the scope of the present paper, the interested reader is referred to [4-7], where many of the theoretical aspects of microwave imaging as well as a variety of practical applications are considered and discussed, and a wide literature is suggested.

Among the various possible configurations of the microwave imaging problem, a scenario that is acquiring an increasing interest is represented by targets enclosed inside conducting enclosures [8,9]. Indeed, this kind of configuration may be useful in systems for biomedical diagnostics [10-13], as well as to inspect the content of pipes [14] or storage containers [15] in industrial processes and agriculture.

On the one hand, the use of conductive enclosures shields the investigation and measurement domains from external interferences and provides well-defined boundary conditions. Moreover, the container bounded by the outer conductor may be filled with proper (liquid or gel) matching media, which are particularily useful in medical applications. On the other hand, the electromagnetic (EM) field is usually strongly perturbed by the presence of the outer conductor, which should be taken into account inside the inverse scattering algorithm.

In this paper, we consider the cylindrical configuration schematized in Figure 1. In particular, we are interested in conductive circular enclosures, which can be part of the tomographic imaging system themselves, as for example it is in some devices for breast or head imaging, or can stand for the boundary of the domain to be imaged, as it could be in the case of monitoring inside pipelines. 


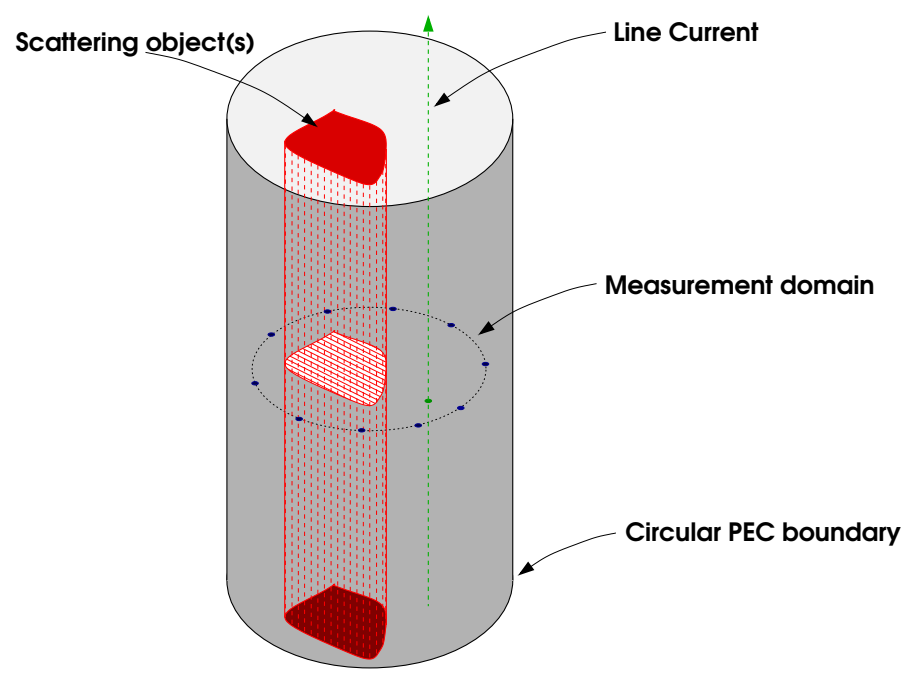

Figure 1. Schematic geometry of the considered problem.

As to the solution of microwave inverse scattering problems, innovative techniques are continuously proposed by the research community [16-22]. It is also worth noting that some specific approaches have been investigated for the imaging inside metallic enclosures, such as eigenfunction-based inversion algorithms [23,24]. Among the various possible inversion schemes, Newton-based methods look promising in many contexts, for their effectiveness in dealing with the intrinsic nonlinearity of the problem at hand [25-27]. Furthermore, it has been shown that facing the problem in non-Hilbertian Lebesgue spaces [28-30] may allow a more accurate reconstruction of the dielectric properties of the imaged domain in various configurations [31,32].

In this paper, we investigate the possibility of exploiting a Newton-based inversion method in non-Hilbertian Lebesgue spaces [33] for the microwave imaging of targets located inside conducting cylinders, illuminated by antennas modeled as line-current sources in the axial direction. In particular, only perfectly electric conducting (PEC) enclosures are taken into account. The adoption of the configuration of Figure 1 reflects in a change of the Green's function and consequently of the kernel of the integral operators used to describe the electromagnetic problem, with non-negligible effects on results. The proposed inversion technique is assessed with a set of numerical simulations in canonical cases, aimed at verifying the dependence of the imaging performance on the size of the enclosure and the loss of the infill dielectric medium, also comparing results with the free space case. Furthermore, for a fixed size of the imaging chamber, a comparison with conventional $L^{2}$ regularization is presented.

The paper is organized as follows. The electromagnetic scattering problem is formulated in Section 2, highlighting the specific issues associated with the presence of a conducting enclosure. Section 3 briefly outlines the structure of the adopted inverse scattering method. Numerical results are described and discussed in Section 4. At the end of the paper, Section 5 draws some concluding considerations.

\section{Problem Formulation}

In this paper, two-dimensional (2D) configurations are assumed, where the geometry is invariant along the $z$ axis. Under $z$-polarized transverse magnetic (TM) illumination conditions, all the involved quantities only depend on the position in the transverse plane, which is indicated by cylindrical coordinates $\rho, \vartheta$ as defined in Figure 2. Time-harmonic electromagnetic fields are assumed with a time dependence $\mathrm{e}^{j \omega t}$. Nonmagnetic materials are considered, characterized by a permeability $\mu=\mu_{0}=4 \pi \times 10^{-7} \mathrm{H} / \mathrm{m}$. The scattering object(s) under test (OUT) are placed inside a circular conducting cylinder of internal radius $a$, which is centered at the axes origin. The cylindrical enclosure is filled by a background medium with a possibly complex dielectric permittivity $\varepsilon_{b}=\varepsilon_{0} \tilde{\varepsilon}_{r, b}=\varepsilon_{0} \varepsilon_{r, b}-j \sigma_{b} / \omega$, where 
$\varepsilon_{0} \simeq 8.85 \times 10^{-12} \mathrm{~F} / \mathrm{m}$ is the vacuum dielectric permittivity, $\varepsilon_{r, b}$ is the relative permittivity, and $\sigma_{b}$ is an (equivalent) background conductivity. Illumination field is provided by an ideal infinite line of current of complex amplitude $I_{z}$ placed at $\left(\rho_{i}, \vartheta_{i}\right)$, i.e.:

$$
\bar{J}(\rho, \vartheta)=\frac{I_{z}}{\rho} \delta\left(\rho-\rho_{i}\right) \delta\left(\vartheta-\vartheta_{i}\right) \hat{z}
$$

where $\frac{1}{\rho} \delta\left(\rho-\rho_{i}\right) \delta\left(\vartheta-\vartheta_{i}\right)$ is the Dirac $\delta$-function for cylindrical coordinates and $z$ invariance [34].

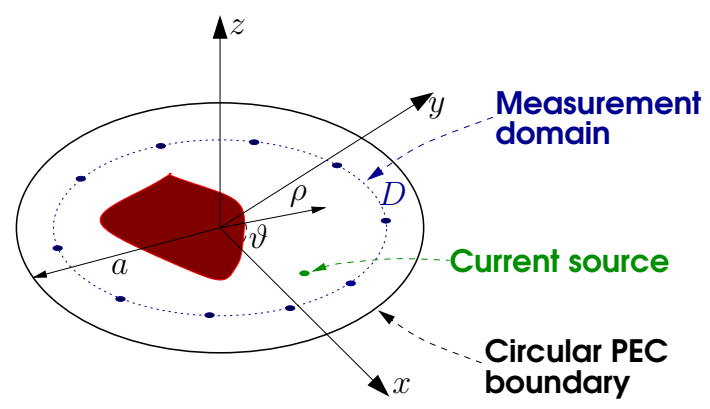

Figure 2. Assonometric projection of the transverse plane, and the related coordinate systems.

Let us denote as $e_{i}(\rho, \theta)$ the $z$ component of the incident electric field inside the cylinder, which is a solution to the scalar Helmholtz equation:

$$
\nabla^{2} e_{i}(\rho, \vartheta)+k_{b}^{2} e_{i}(\rho, \vartheta)=j \omega \mu_{0} \frac{I_{z}}{\rho} \delta\left(\rho-\rho_{i}\right) \delta\left(\vartheta-\vartheta_{i}\right)
$$

where $k_{b}=\omega \sqrt{\mu_{0} \varepsilon_{b}}$ is the wavenumber, subject to the boundary condition $e_{i}(a, \theta)=0$.

If some scattering object is present inside the cylinder, the (z-directed) total electric field $e_{t}(\rho, \theta)$ can always be written as the sum:

$$
e_{t}(\rho, \theta)=e_{i}(\rho, \theta)+e_{s}(\rho, \theta)
$$

where $e_{s}(\rho, \theta)$ is the (z-directed) scattered field.

According to the volume equivalence theorem [35], the scattering field can be expressed in an integral form

$$
e_{s}(\rho, \theta)=-k_{b}^{2} \int_{D} \tau\left(\rho^{\prime}, \vartheta^{\prime}\right) e_{t}\left(\rho^{\prime}, \vartheta^{\prime}\right) g\left(\rho, \vartheta ; \rho^{\prime}, \vartheta^{\prime}\right) \mathrm{d} \rho^{\prime} \mathrm{d} \vartheta^{\prime}
$$

where $D$ is the considered investigation domain (which as reported in Figure 2, is a circular region located in the transverse plane) and

$$
\tau(\rho, \vartheta)=\frac{\tilde{\varepsilon}_{r}(\rho, \vartheta)}{\tilde{\varepsilon}_{r, b}}-1
$$

is the so-called object function, accounting for the dielectric properties of the scatterers by means of the space-dependent complex relative dielectric permittivity $\tilde{\varepsilon}_{r}(\rho, \vartheta)$, and finally $g\left(\rho, \vartheta ; \rho^{\prime}, \vartheta^{\prime}\right)$ is the Green function for the problem.

\section{Green's Function of the Considered Problem}

The Green's function inside a circular PEC cylinder of radius $a$ (Figure 1) is the solution of the following Helmholtz equation:

$$
\nabla^{2} g\left(\rho, \vartheta ; \rho^{\prime}, \vartheta^{\prime}\right)+k_{b}^{2} g\left(\rho, \vartheta ; \rho^{\prime}, \vartheta^{\prime}\right)=\frac{1}{\rho} \delta\left(\rho-\rho^{\prime}\right) \delta\left(\vartheta-\vartheta^{\prime}\right)
$$


subject to the boundary condition $g\left(a, \vartheta ; \rho^{\prime}, \vartheta^{\prime}\right)=0$.

The solution to this equation is discussed in many articles and textbooks (see, for example [35-38]), and can be expressed in some different forms. In this work the following expression will be used [35]:

$$
g\left(\rho, \vartheta ; \rho^{\prime}, \vartheta^{\prime}\right)=-\frac{1}{4} \begin{cases}\sum_{n=-\infty}^{+\infty}\left[J_{n}\left(k_{b} \rho^{\prime}\right) Y_{n}\left(k_{b} a\right)-J_{n}\left(k_{b} a\right) Y_{n}\left(k_{b} \rho^{\prime}\right)\right] \times & \\ \times \frac{J_{n}\left(k_{b} \rho\right)}{J_{n}\left(k_{b} a\right)} \mathrm{e}^{j n\left(\vartheta-\vartheta^{\prime}\right)} & \rho<\rho^{\prime} \\ \sum_{n=-\infty}^{+\infty}\left[J_{n}\left(k_{b} \rho\right) Y_{n}\left(k_{b} a\right)-J_{n}\left(k_{b} a\right) Y_{n}\left(k_{b} \rho\right)\right] \times & \\ \times \frac{J_{n}\left(k_{b} \rho^{\prime}\right)}{J_{n}\left(k_{b} a\right)} \mathrm{e}^{j n\left(\vartheta-\vartheta^{\prime}\right)} & \rho>\rho^{\prime}\end{cases}
$$

where $J_{n}$ and $Y_{n}$ are the Bessel functions of the first and of the second kind, respectively, of order $n$. Without going in too much theoretical details (which were the subject of other works, such as [8]) here we want just to stress two aspects of the problem, making it much more tricky than the analogous in free-space:

- while in free space the scattering field can be expanded into a simple sum of progressing waves, in the present problem the solution is made by a sum of complicated standing waves, and many resonant modes can arise inside the cavity;

- the incident field is also strongly affected by the cavity boundaries: while the line current produces a simple circular wave in free space, in the present problem the incident field has the same form of the Green's function (compare Equations (2) and (6)), hence it contains many contributions, made of standing waves depending on the cavity dimensions.

In (7), the presence of the term $J_{n}\left(k_{b} a\right)$ causes $g$ to assume high values when it approaches zero (in particular, $g \rightarrow \infty$ when $J_{n}\left(k_{b} a\right)=0$ ). In the lossless case, the positions of $\mathrm{TM}_{n l}$ resonances can be found based on the $l$-th root of the Bessel function of first kind with order $n$.

Investigation about the zeros of Bessel's functions is a problem that is not new in physics and the first comprehensive works about this topic date back the second half of the 19th century [39,40]. Many of these and other findings (e.g., [41]) about Bessel's functions are collected and extended in the monumental book by Watson [42]. Further studies were since then carried out and this topic is presently still an open research field for mathematics. A complete review of the literature about Bessel's functions is outside the scope of the present paper; however we refer the reader to some papers [43-53], containing relevant results for the problem faced in the present work. It is worth nothing that also some works, aimed at other goals, contain notable insight about the zeros of Bessel's functions [54-58].

In particular, in order to underline the behavior of resonances within the enclosure, some properties of the zeros of Bessel's functions are worth recalling. Please note that such properties can be valid for any real order $v$, but we focus on integer order $n$. Let $\zeta_{n, l}$ denote the $l$-th $(l \geq 1)$ zero of the Bessel function of first kind with order $n$. Then:

$$
\begin{array}{ll}
\text { a. } & \lim _{n \rightarrow \infty}\left(\zeta_{n, l+1}-\zeta_{n, l}\right)=\pi \\
\text { b. } & \zeta_{n, 1}<\zeta_{n+1,1}<\zeta_{n, 2}<\zeta_{n+1,2}<\zeta_{n, 3}<\ldots ; \\
\text { c. } & \zeta_{n, l}>n+l \pi+\frac{\pi}{2}-\frac{1}{2} \\
\text { d. } & \zeta_{n, l}<\left(l+\frac{n}{2}-\frac{0.965}{4}\right) \pi-\frac{n^{2}}{2}\left[\left(l+\frac{n}{2}-\frac{0.965}{4}\right) \pi\right]^{-1} n>0 ; \\
\text { e. } & \zeta_{n, 1}<2(n+1)(n+5)(5 n+11) /(7 n+19) \quad n>-1 .
\end{array}
$$


The first property states that, for each $n$, the zeros approaches a regular distribution, in accordance with the asymptotic behavior of $J_{n}$. The second statement is about the interlacing property of the zeros. This should explain how the zeros of Bessel's functions of any order tend to distribute. In particular, it should be stressed that between two consecutive zeros of $J_{n}$ lies exactly one zero of $J_{n+1}$. Furthermore, property (c) provides a lower bound for the zeros, and it can be seen that "zeros are strictly increasing functions of both index and order" [58]. Therefore, properties $(a),(b)$, and (c) show that the number of zeros in an interval $(x-\Delta, x+\Delta)$ strictly increases as $x$ becomes larger.

The number of zeros in an interval could also be estimated by using upper bounds that exists for the zeros of Bessel functions. Properties (e) and (d) are about two simple upper bounds. More sharp (and complicated) bounds can also be found in the literature (e.g., $[45,47,48,59,60])$; however, for the problem faced in the present work, the number of zeros is small enough to allow computing to a high accuracy the position of each zero.

Previous analysis is strictly related to the problem dealt with in the present work, since, as the radius of the enclosure increases, the number of possible resonances increases, too, and their distribution follows the behavior of Bessel zeros. As an example, zeros corresponding to the resonances of a conducting enclosure filled with vacuum and with radius $a$, ranging between 0 and $3 \lambda$, have been evaluated, and their number have been computed in each of the intervals $(x-\Delta, x+\Delta), \Delta=0.25 \lambda, x=[0.25+m / 2] \lambda, m=0, \ldots, 5$. In Figure 3 the histogram of the results is shown. As can be seen, the predicted behavior is perfectly confirmed. For convenience, the first $\mathrm{TM}_{n l}$ resonances versus the radius of the PEC cylinder (up to $3 \lambda$ ) are reported in Table 1.

Table 1. First $\mathrm{TM}_{n l}$ resonances versus the radius of the conducting enclosure (for radiuses up to $3 \lambda$ ).

\begin{tabular}{|c|c|c|c|c|c|}
\hline Order, $n$ & Root, $l$ & Radius, $a / \lambda$ & Order, $n$ & Root, $l$ & Radius, $a / \lambda$ \\
\hline 0 & 1 & 0.382565575636716 & 9 & 1 & 2.12443502732519 \\
\hline 1 & 1 & 0.609557227746547 & 6 & 2 & 2.16181776676093 \\
\hline 2 & 1 & 0.816987450864820 & 4 & 3 & 2.28641855021103 \\
\hline 0 & 2 & 0.878147628239934 & 10 & 1 & 2.30279831942887 \\
\hline 3 & 1 & 1.01497187625880 & 2 & 4 & 2.35377646939679 \\
\hline 1 & 2 & 1.11605681508930 & 7 & 2 & 2.35780395133906 \\
\hline 4 & 1 & 1.20717221350811 & 0 & 5 & 2.37524718150666 \\
\hline 2 & 2 & 1.33903593944348 & 11 & 1 & 2.48007141762091 \\
\hline 0 & 3 & 1.37665636071269 & 5 & 3 & 2.49762238062566 \\
\hline 5 & 1 & 1.39538925994123 & 8 & 2 & 2.55132863821835 \\
\hline 3 & 2 & 1.55280761241691 & 3 & 4 & 2.58086897429352 \\
\hline 6 & 1 & 1.58066078745604 & 1 & 5 & 2.62018841505947 \\
\hline 1 & 3 & 1.61842038016315 & 12 & 1 & 2.65639874693837 \\
\hline 4 & 2 & 1.76020125084557 & 6 & 3 & 2.70500953319621 \\
\hline 7 & 1 & 1.76364706145171 & 9 & 2 & 2.74277582388405 \\
\hline 2 & 3 & 1.84851296702361 & 4 & 4 & 2.80239128808070 \\
\hline 0 & 4 & 1.87582635499932 & 13 & 1 & 2.83189617009082 \\
\hline 8 & 1 & 1.94479780216248 & 2 & 5 & 2.85709234145654 \\
\hline 5 & 2 & 1.96285556025129 & 0 & 6 & 2.87478938633534 \\
\hline 3 & 3 & 2.07049020258037 & 7 & 3 & 2.90923374085231 \\
\hline 1 & 4 & 2.11956574517874 & 10 & 2 & 2.93244082347851 \\
\hline
\end{tabular}




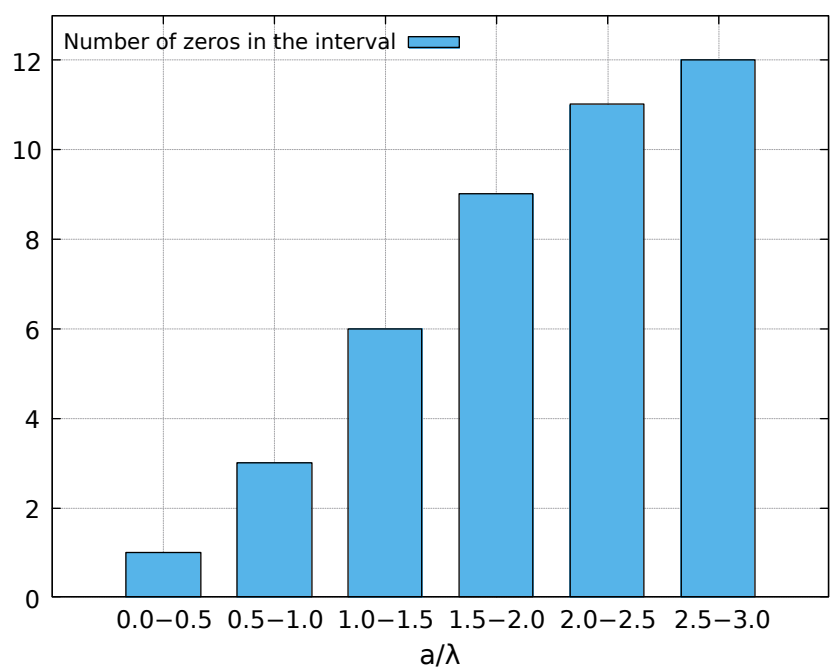

Figure 3. Number of resonances for an enclosure as the radius ranges from 0 to $3 \lambda$.

When the argument of a Bessel function becomes complex, as is in the case of conductive media, the behavior changes in accordance to the variation on the imaginary part $\Im\{z\}$ of the argument $z=x-j y$. In particular, while $J_{n}(x)$ is bounded, this is no longer the case for $J_{n}(x-j y)$, where both the real and the imaginary parts tend to increase as the argument increases. An in-depth discussion about Bessel's function of complex arguments is outside the scope of the present work and we refer interested readers to some specialized literature $[61,62]$. However, to investigate some practical case, let us consider:

$$
z=x-j y=k_{b} \rho=\left[\Re\left\{k_{b}\right\}-j \Im\left\{k_{b}\right\}\right] \rho
$$

where $k_{b}$ is complex, while $\rho$ is real. In particular, two effects can be observed: the behavior with respect to lossless case gets smoothed, since there are no longer zeros, and the amplitude of $J_{n}\left(k_{b} \rho\right)$ tends to oscillate and decrease up to a certain value of $\rho$. For larger values, oscillations are quickly damped and $\left|J_{n}\left(k_{b} \rho\right)\right|$ begins to increase and eventually approaches infinity. In Figure 4 an example is shown, for different orders of Bessel functions and for different values of $\sigma_{b}$. In particular, in the upper left corner, the function giving the larger number of zeros, namely $J_{0}$, is plotted, while in lower right corner we have shown the behavior of $J_{13}$, being 13 the maximum order for which it is possible having a zero in the considered range. The other two graphs, referring respectively to $J_{3}$ and $J_{7}$, are intended to give an idea of the trend, as the order of the Bessel function varies. From the example in Figure 4 it can be deduced that, while in general, a conductive background would be an obstacle to the solution of an inverse scattering problem, for a certain range of values of $\sigma_{b}$ (we could consider $\sigma_{b} \lesssim 3 \mathrm{mS} / \mathrm{m}$ in the example, with $\Im\left\{k_{b}\right\} \lesssim 0.15 \Re\left\{k_{b}\right\}$ ) an advantage can come from the damping of the resonances, while the losses can still be managed. 

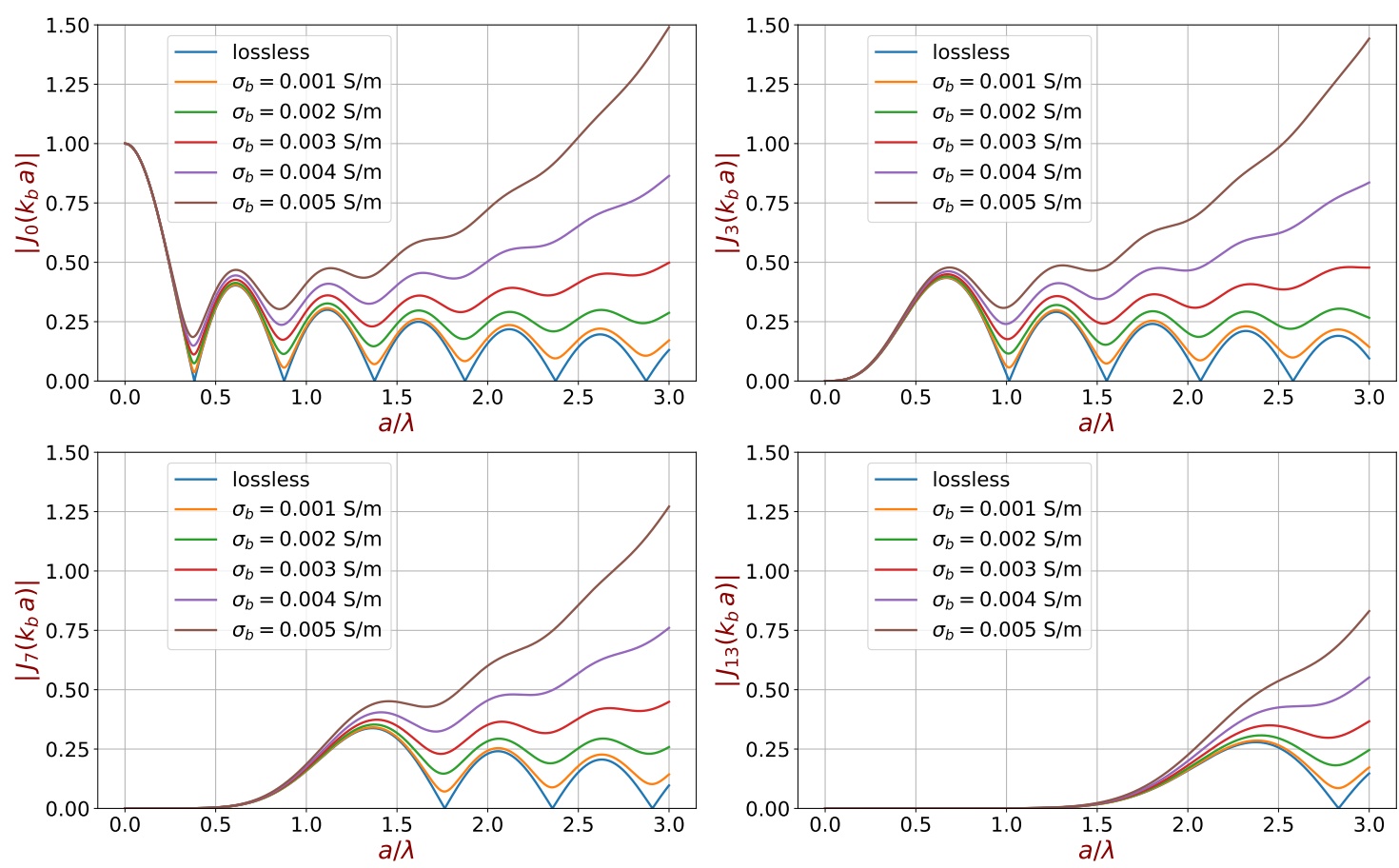

Figure 4. Behavior of the magnitude of different Bessel's functions $J_{n}\left(k_{b} a\right)$ for a background with $\varepsilon_{b}=\varepsilon_{0}-j \sigma_{b} / \omega$ and different values of $\sigma_{b}$, versus the radius of PEC cylinder $a$. In the upper left corner $J_{0}$ is shown.

\section{Nonlinear Inverse Scattering Method}

In solving the inverse problem, the goal is to retrieve the object function $\tau(\rho, \vartheta)$ inside an investigation domain $D$ starting from a set of measurements of the scattered field $e_{s}(\rho, \theta)$ collected in an observation domain $O$. Based on (4), it can be shown that $\tau(\rho, \vartheta)$ is connected to the measured scattered electric field $e_{S}(\rho, \theta)$ by means of a nonlinear relationship:

$$
e_{S}(\rho, \theta)=\mathcal{A}_{O} \tau\left(I-\mathcal{A}_{D} \tau\right)^{-1} e_{i}(\rho, \theta)=\mathcal{N}(\tau)(\rho, \theta), \quad(\rho, \theta) \in O
$$

where the linear operators $\mathcal{A}_{\{O, D\}}$ are given by

$$
\mathcal{A}_{\{O, D\}} x(\rho, \theta)=-k_{b}^{2} \int_{D} x\left(\rho^{\prime}, \vartheta^{\prime}\right) g\left(\rho, \vartheta ; \rho^{\prime}, \vartheta^{\prime}\right) \mathrm{d} \rho^{\prime} \mathrm{d} \vartheta^{\prime}, \quad(\rho, \theta) \in\{O, D\}
$$

The inverse scattering method is characterized by an inexact-Newton scheme, where (9) is iteratively linearized around the reconstructed value of the object function $\tau_{i}(\rho, \vartheta)$ at the $i$-th step $(i=1, \ldots, I)$. Starting the iterations with an initial value $\tau_{1}=0$, the result of such a linearization is the equation

$$
\mathcal{N}_{i}^{\prime} \delta=\underbrace{e_{S}-\mathcal{N}\left(\tau_{i}\right)}_{b_{i}}
$$

where $\delta \in A, b_{i} \in B$ and $\mathcal{N}_{i}^{\prime}: A \rightarrow B$ is the Fréchet derivative of the nonlinear operator $\mathcal{N}$ calculated at $\tau_{i}$. The solution of (11) in the unknown $\delta$ is again performed by an iterative loop, which is a truncated Landweber-like method that considers $A, B$ as Lebesgue spaces $L^{p}$ [33]. This regularized solution approach, at the $k$-th step $(k=1, \ldots, K)$, has been implemented as

$$
\delta_{k+1}=J_{A^{*}}\left[J_{A}\left(\delta_{k}\right)-\gamma \mathcal{N}_{i}^{\prime *} J_{B}\left(\mathcal{N}_{i}^{\prime} \delta_{k}-b_{i}\right)\right]
$$

where $J_{A}(\cdot), J_{A^{*}}(\cdot)$ and $J_{B}(\cdot)$ are defined as the duality maps of spaces $A, A^{*}$ (i.e., the dual space of $A$ ) and $B$ [31]; $\mathcal{N}_{i}^{\prime *}$ is the adjoint of $\mathcal{N}_{i}^{\prime} ; \gamma$ is a real positive number. The loop starts with $\delta_{1}=0$. Once (11) is solved by means of iterations (12), a regularized version of the unknown of the linear problem $\tilde{\delta}$ is available. Then, the object function inside the investigation domain is updated as 


$$
\tau_{i+1}(\rho, \theta)=\tau_{i}(\rho, \theta)+\tilde{\delta}(\rho, \theta), \quad(\rho, \theta) \in D
$$

and a new linearization is performed, until convergence is reached.

\section{Results of Numerical Simulations}

The behavior of the proposed inverse scattering technique when operating in conducting enclosures has been analyzed from a numerical point of view in canonical configurations, involving circular dielectric cylinders.

The simulations have been oriented at understanding the influence of the external conducting cylinder on the reconstruction performance. To this end, two distinct cases have been considered, regarding a small and a large enclosure. The difference between these two cases is related to the definition of the investigation domain $D$ and the observation domain $O$. This distinction has also been made to follow, in the case of sufficiently large enclosures, the guidelines about the position of the observation domain with respect to the conducting boundary provided in [8]. A background characterized by $\varepsilon_{b}=\varepsilon_{0}-j \sigma_{b} / \omega$ has been considered. The electric field data have been simulated at the angular frequency $\omega=2 \pi f$, with $f=300 \mathrm{MHz}$, by means of a custom numerical code based on the method of moments, and corrupted with an additive Gaussian noise with zero mean value and signal-to-noise ratio equal to $20 \mathrm{~dB}$. In all cases, the inversion method has been run with the same parameters in order to compare results. In particular, a Lebesgue space exponent $p=1.2$ is considered; the iterative loops have been stopped when a maximum number of inexact-Newton iterations $I=10$ and a maximum number of Landweber steps $K=100$ are reached, or when the relative variation of the data residual falls below a threshold value $r_{\text {th }}=1 \%$.

The results have been evaluated from a quantitative viewpoint by means of the normalized reconstruction error (NRE)

$$
N R E=\frac{\left\|\tau-\tau_{a c t}\right\|}{\left\|\tau_{a c t}\right\|}
$$

For each analyzed case, $N=15$ simulations with different random noise have been executed, calculating the mean value and the standard deviation of the obtained values of the NRE.

\subsection{Small Conducting Enclosure}

In the case of the small conducting enclosure, the radius of the outer PEC cylinder has been varied in the range $a \in[0.25,1.025] \lambda$ with $0.025 \lambda$ steps. A set of $S=30$ positions, equally spaced on a circumference with radius $\rho_{s}=a-0.1 \lambda$, have been defined to host both sources and measurement points in a multistatic and multiview configuration (i.e., one of the position at a time is occupied by a source antenna, whereas all the other ones are used to sample the scattered electric field).

The investigation domain is a circular region centered at the origin with diameter $d_{D}=0.25 \lambda$. This region contains a circular dielectric cylinder of diameter $d_{c}=0.125 \lambda$, centered at $\left(\rho_{c}, \theta_{c}\right)=(0.05 \lambda, 0)$, characterized by a relative dielectric permittivity $\varepsilon_{r, c}=2$ and electric conductivity $\sigma_{c}=10 \mathrm{mS} / \mathrm{m}$. For the forward problem solution, $D$ has been subdivided into $N_{f}=1976$ square subdomains with side length equal to $l_{s f}=0.005 \lambda$. Conversely, a discretization with $N_{i}=1264$ cells of side $l_{s i}=0.00625 \lambda$ has been adopted inside the inversion procedure.

Figure 5 reports the NRE reconstruction error versus the size of the enclosure $a$, for three different values of background loss $\sigma_{b}=\{0,1,10\} \mathrm{mS} / \mathrm{m}$ (which correspond to loss $\operatorname{tangents} \tan \delta=\{0,0.06,0.6\}$ ). Some examples of reconstructed dielectric permittivity are also shown in Figures 6 and 7 , along with the magnitude of the Green's function inside the conducting cylinder for a source at $\left(\rho_{s}, 0\right)$. All results are compared with the free space case, where the same configuration but without the PEC enclosure have been simulated. 


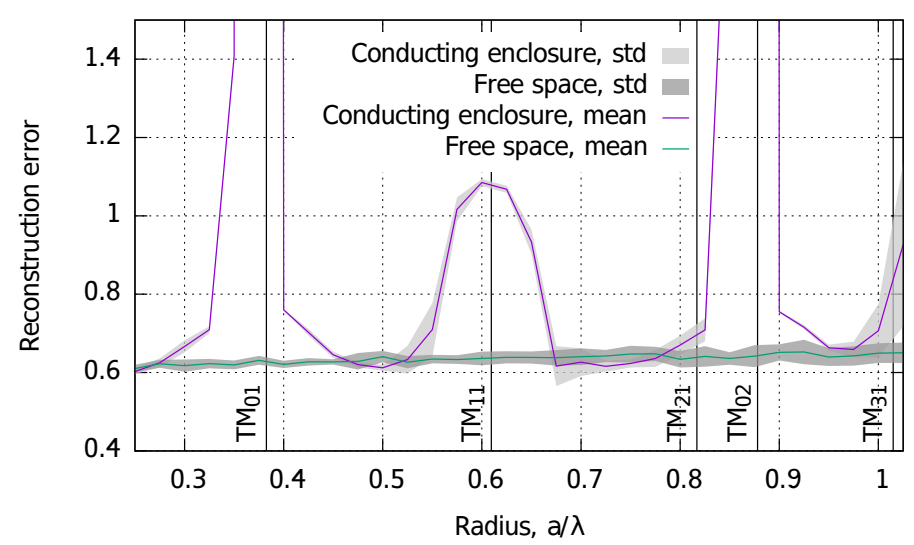

(a)

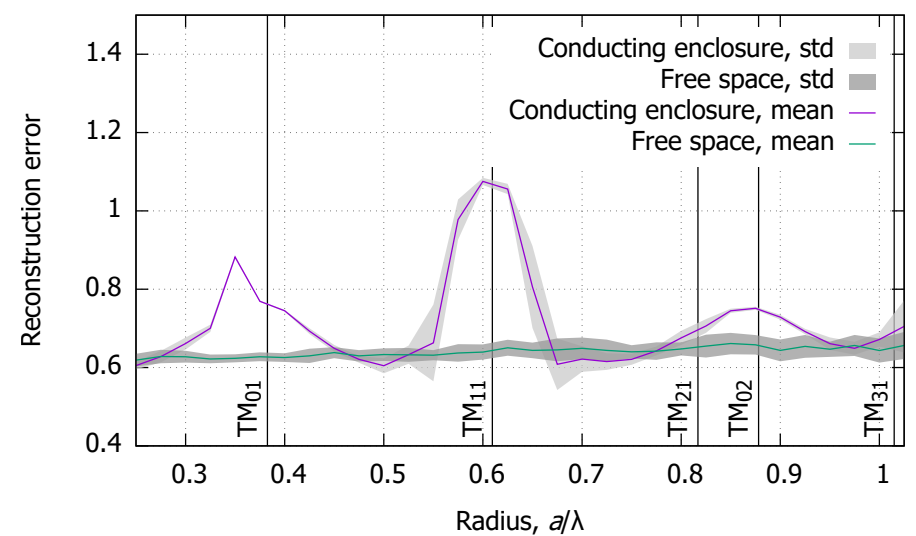

(b)

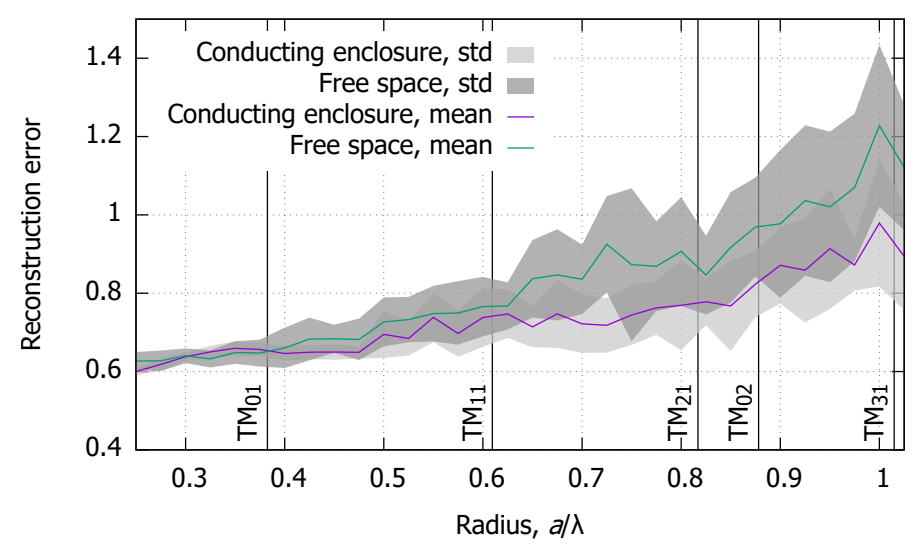

(c)

Figure 5. Small conducting enclosure. Reconstruction error versus the radius of the conducting enclosure, for three values of the background conductivity: (a) lossless, $\sigma_{b}=0 \mathrm{~S} / \mathrm{m}$; (b) $\sigma_{b}=1 \mathrm{mS} / \mathrm{m}$; (c) $\sigma_{b}=10 \mathrm{mS} / \mathrm{m}$. 


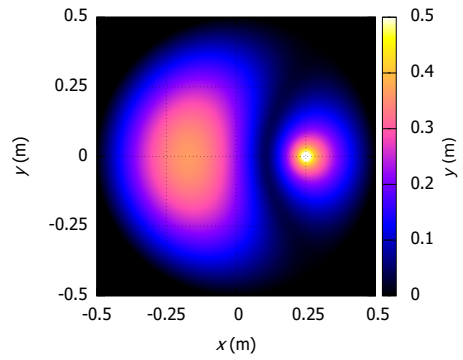

(a)

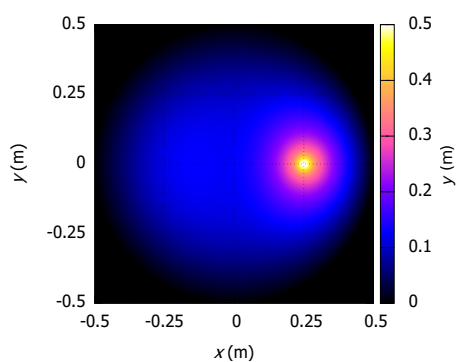

(d)

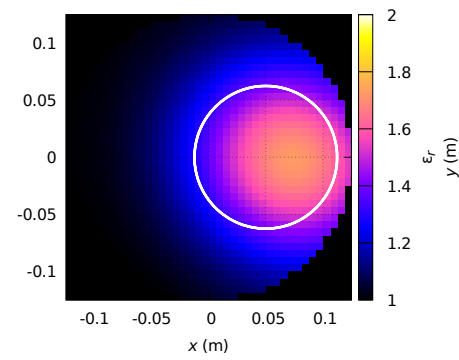

(b)

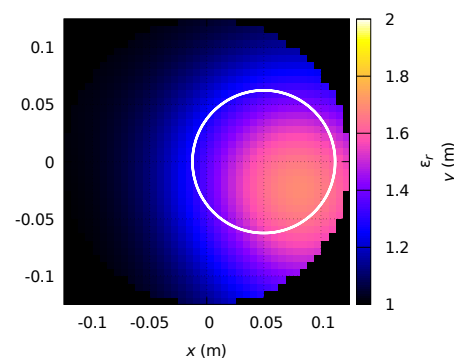

(e)

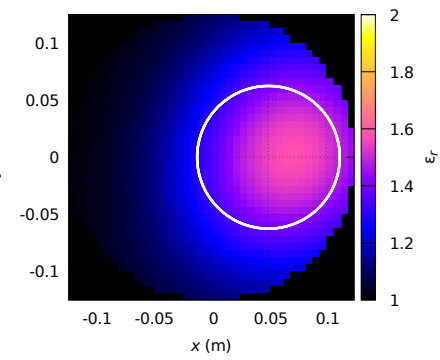

(c)

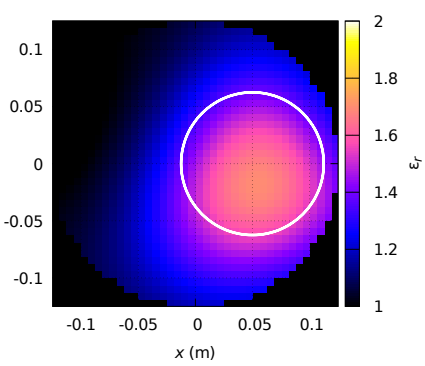

(f)

Figure 6. Examples of Green's functions and reconstructed distributions of the relative dielectric permittivity with small conducting enclosure, $a=\lambda / 2$. Upper row: results with $\sigma_{b}=1 \mathrm{mS} / \mathrm{m}$ : (a) Green's function magnitude for a source at $\left(\rho_{s}, 0\right)$; (b) Reconstruction with cylindrical enclosure; (c) Reconstruction in free space. Lower row: results with $\sigma_{b}=10 \mathrm{mS} / \mathrm{m}$ : (d) Green's function for point source located at $\left(\rho_{s}, 0\right)$; (e) Reconstruction with cylindrical enclosure; (f) Reconstruction in free space.

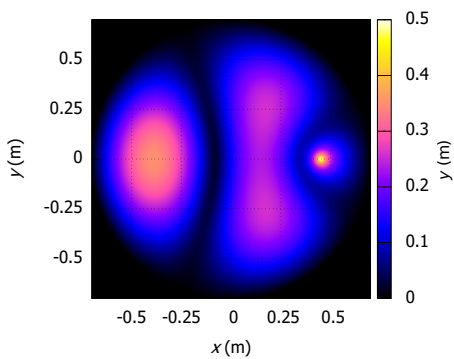

(a)

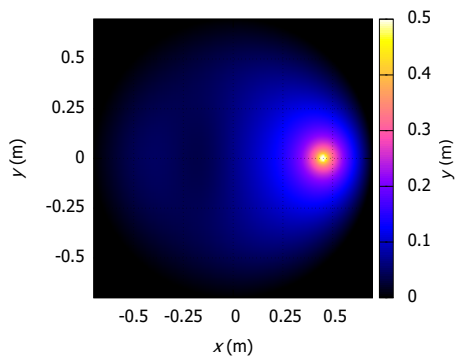

(d)

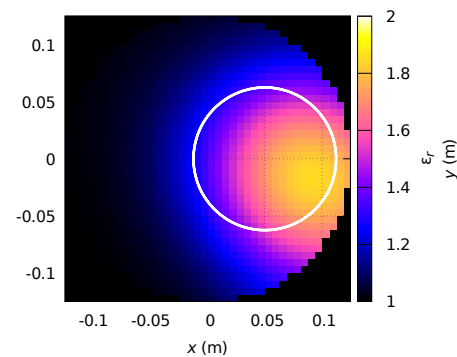

(b)

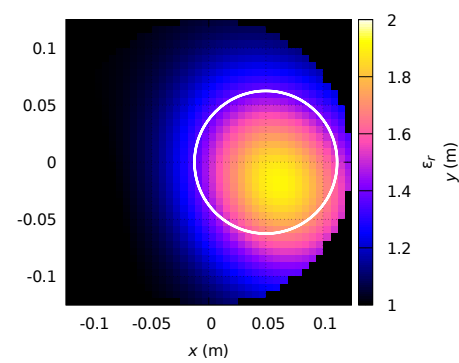

(e)

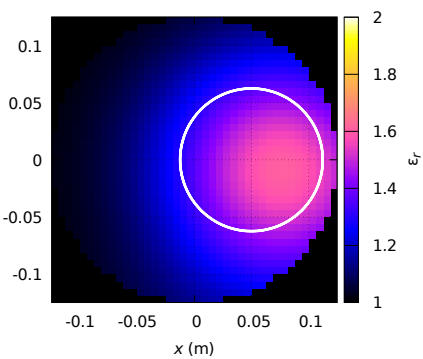

(c)

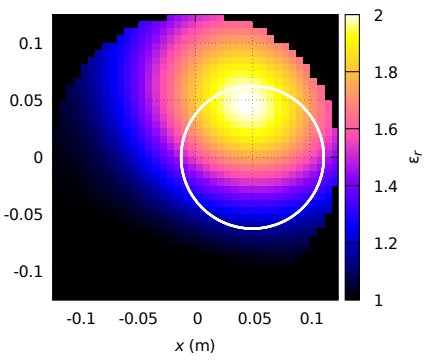

(f)

Figure 7. Examples of Green's functions and reconstructed distributions of the relative dielectric permittivity with small conducting enclosure, $a=0.7 \lambda$. Upper row: results with $\sigma_{b}=1 \mathrm{mS} / \mathrm{m}$ : (a) Green's function magnitude for a source at $\left(\rho_{s}, 0\right)$; (b) Reconstruction with cylindrical enclosure; (c) Reconstruction in free space. Lower row: results with $\sigma_{b}=10 \mathrm{mS} / \mathrm{m}$ : (d) Green's function for point source located at $\left(\rho_{s}, 0\right)$; (e) Reconstruction with cylindrical enclosure; (f) Reconstruction in free space. 
As reported in the graphs, five different resonances may arise with this range of radiuses of the PEC cylinder. For small or absent background losses, the $\mathrm{TM}_{01}, \mathrm{TM}_{11}$ and $\mathrm{TM}_{02}$ modes clearly cause a significant degradation of results, impairing a correct reconstruction of the inside dielectric cylinder. In the middle points between these significant resonances, results are comparable or even slightly better (in average) than the free space ones. When background losses rise, a completely different trend is observed (Figure 5c): there are no points with higher errors due to resonances, and the average of NRE demonstrates some advantages in adopting a conducting enclosure with respect to the free space conditions. Clearly, as expected, errors in both situations increase by enlarging the radius $a$, since this also increases the distance between the target and the observation domain $O$. However, the error increase with the surrounding metallic enclosure is slightly less steep than the free space case.

\subsection{Large Conducting Enclosure}

In the case of large conducting enclosure, the radius of the PEC cylinder has been varied in the range $a \in[1.0,3.0] \lambda$ with $0.125 \lambda$ steps. The same number of $S=30$ positions as before has been used, but this time they are located on a circumference with radius $\rho_{s}=a-0.25 \lambda$. A multistatic and multiview configuration has been always considered. The investigation domain is larger than the previous case, and is a circular region centered at the origin with diameter $d_{D}=\lambda$. A circular dielectric cylinder characterized by the same dielectric properties as in Section 4.1, but with diameter $d_{c}=0.2 \lambda$ and centered at $\left(\rho_{c}, \theta_{c}\right)=(0.2 \lambda, 0)$ is placed inside the PEC enclosure.

The forward electromagnetic problem has been solved by subdividing $D$ into $N_{f}=1976$ square subdomains with side length $l_{s f}=0.02 \lambda$, whereas a discretization with $N_{i}=1264$ cells of dimension $l_{s i}=0.025 \lambda$ has been used for the inverse problem'solution.

The mean value and standard deviation of the NRE in this case is shown in Figure 8, which reports the reconstruction errors versus the radius $a$ for $\sigma_{b}=\{0,1,2\} \mathrm{mS} / \mathrm{m}$ $(\tan \delta=\{0,0.06,0.12\})$. Figure 9 shows some examples of the reconstructed relative dielectric permittivity inside $D$ and the Green's function magnitude for a source located at $\left(\rho_{s}, 0\right)$. As can be noticed looking at Table 1 , the number of resonances of the circular cavity dramatically increases for $a>\lambda$. This fact determines the significantly higher errors compared to the corresponding free space cases when no or low background loss is present. It is actually very difficult, even with a practical construction of a metallic chamber in mind, to avoid all the possible resonances. However, by increasing the background loss (e.g., Figure $8 \mathrm{~b}$ ) it can be observed that not all resonances are critical in the same way. Those that cause a worst degradation of reconstruction results are again related to the $\mathrm{TM}_{0 l}$ modes, with $l=\{2,3,4,5\}$, and can be seen in the high-error peaks at $a=\{1.375,1.875,2.375,2.875\} \lambda$. Moreover, similarly to the case of the small enclosure, the $\mathrm{TM}_{1 l}$ resonances have a smaller but yet significant impact. The remaining ones are sufficiently damped by the small background loss and do not produce increases in the $N R E$ in neighboring sizes of the PEC cylinder. Of course, the bigger the cylinder radius, the greater is the effect of background losses.

Moreover, Figure $8 \mathrm{c}$ evidences some interesting facts related to the imaging in conducting enclosures versus their free space counterpart. In the lossy case, reconstruction results obtained inside conducting cylinders are always better then the free space ones, having a smaller mean NRE and even a significantly reduced standard deviation. This is also observed in Figures 9 and 10, where it can be seen that the free space reconstruction have more background artifacts than those obtained in conducting enclosures. In addition, the advantages of embedding the measurement configuration in a metallic cylinder appear more evident if the background losses rise. 


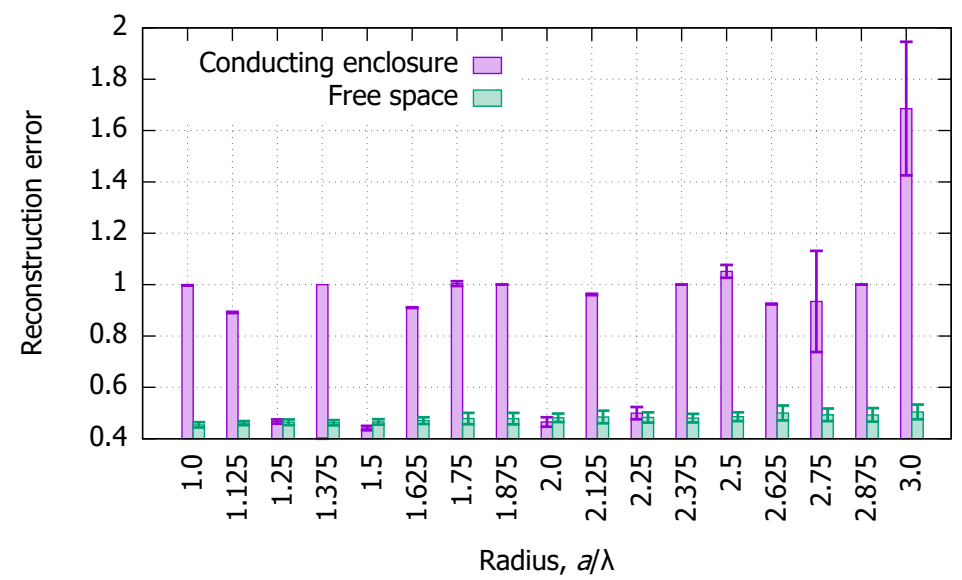

(a)

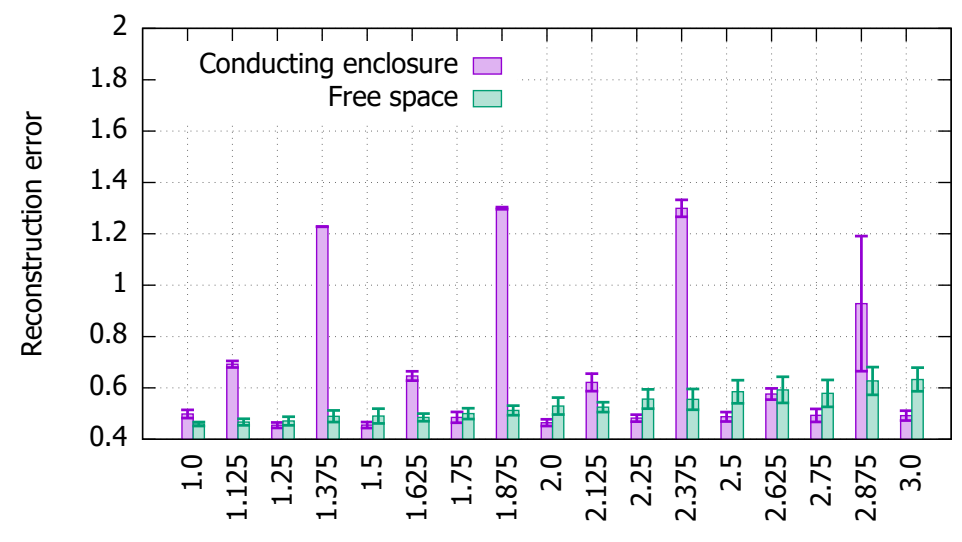

Radius, $a / \lambda$

(b)

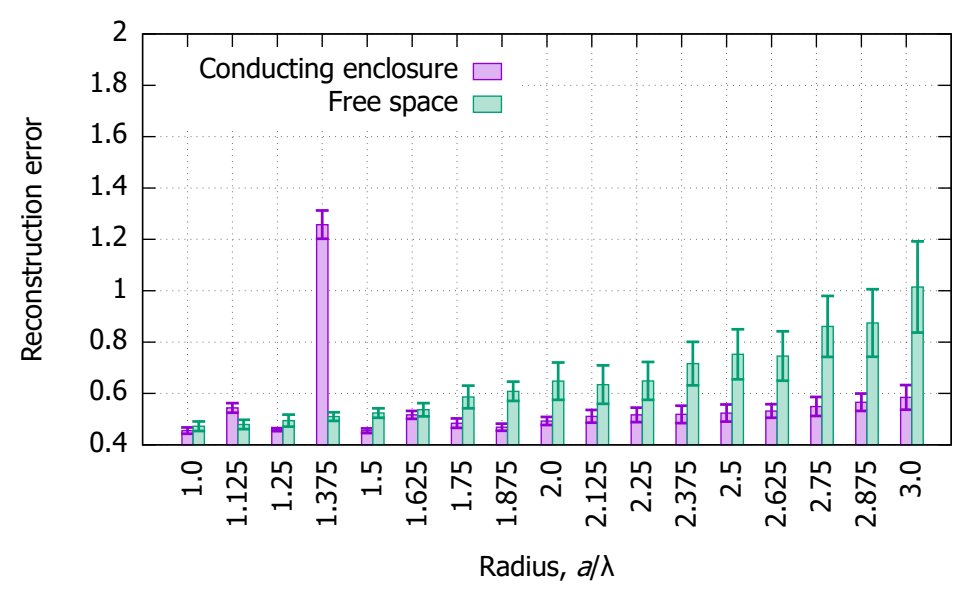

(c)

Figure 8. Large conducting enclosure. Reconstruction error versus the radius of the conducting enclosure: (a) $\sigma_{b}=0 \mathrm{~S} / \mathrm{m}$; (b) $\sigma_{b}=1 \mathrm{mS} / \mathrm{m} ;$ (c) $\sigma_{b}=2 \mathrm{mS} / \mathrm{m}$. 


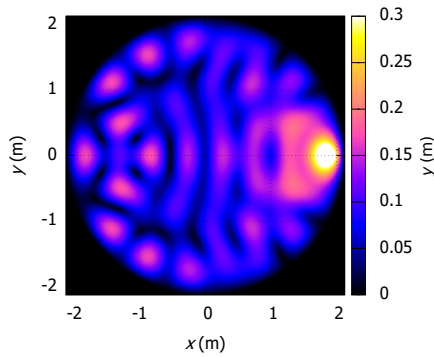

(a)

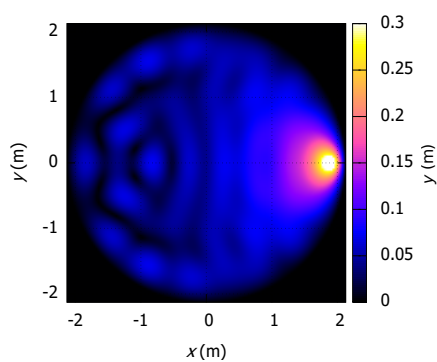

(d)

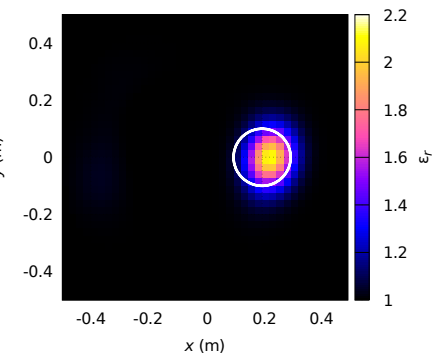

(b)

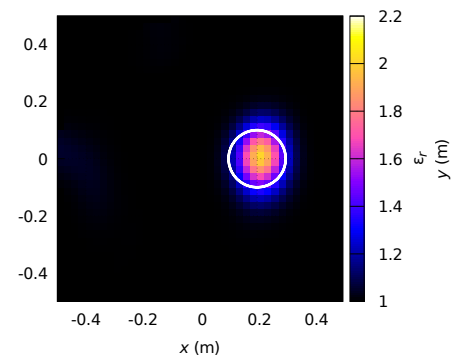

(e)

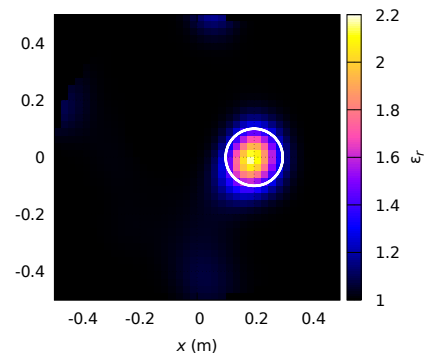

(c)

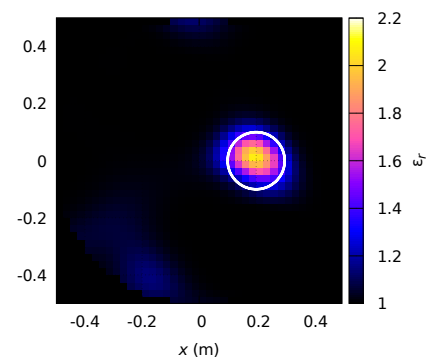

(f)

Figure 9. Examples of Green's functions and reconstructed distributions of the relative dielectric permittivity with small conducting enclosure, $a=2.125 \lambda$. Upper row: results with $\sigma_{b}=1 \mathrm{mS} / \mathrm{m}$ : (a) Green's function magnitude for a source at $\left(\rho_{s}, 0\right)$; (b) Reconstruction with cylindrical enclosure; (c) Reconstruction in free space. Lower row: results with $\sigma_{b}=2 \mathrm{mS} / \mathrm{m}$ : (d) Green's function for point source located at $\left(\rho_{s}, 0\right)$; (e) Reconstruction with cylindrical enclosure; (f) Reconstruction in free space.

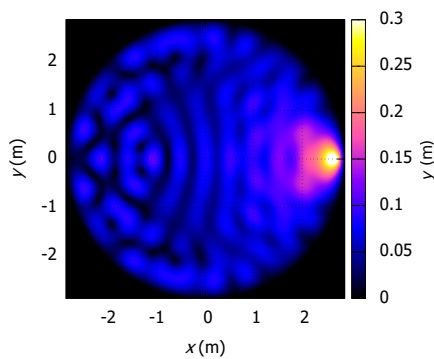

(a)

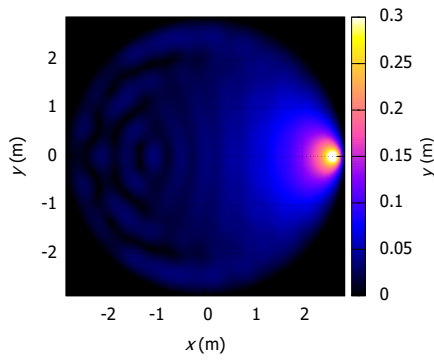

(d)

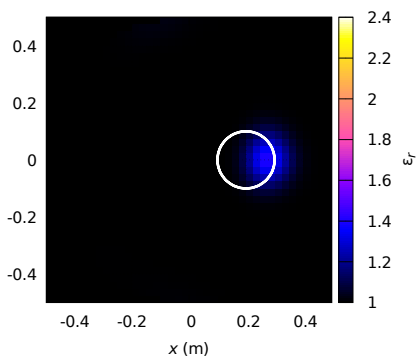

(b)

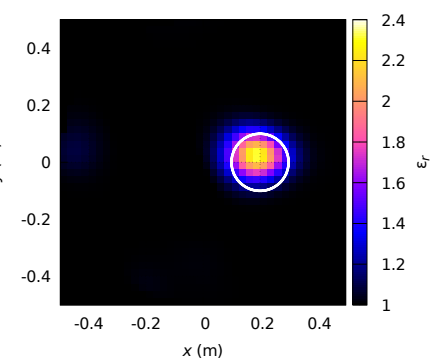

(e)

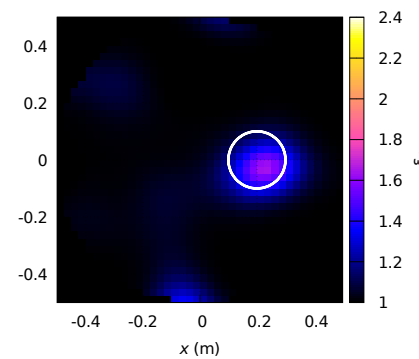

(c)

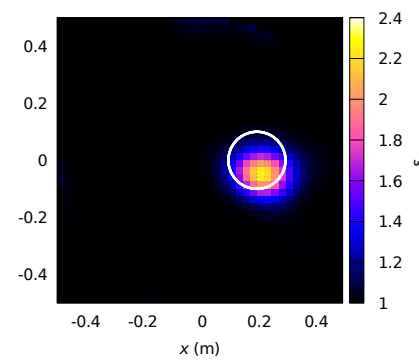

(f)

Figure 10. Examples of Green's functions and reconstructed distributions of the relative dielectric permittivity with small conducting enclosure, $a=2.875 \lambda$. Upper row: results with $\sigma_{b}=1 \mathrm{mS} / \mathrm{m}$ : (a) Green's function magnitude for a source at $\left(\rho_{s}, 0\right)$; (b) Reconstruction with cylindrical enclosure; (c) Reconstruction in free space. Lower row: results with $\sigma_{b}=2 \mathrm{mS} / \mathrm{m}$ : (d) Green's function for point source located at $\left(\rho_{s}, 0\right)$; (e) Reconstruction with cylindrical enclosure; (f) Reconstruction in free space. 
In order to verify how such considerations generalize, additional tests with a different target have been carried out. In particular, a fixed imaging chamber size $a=2.125 \lambda$, with $\sigma_{b}=2 \mathrm{mS} / \mathrm{m}$ and all the other parameters as before has been considered. This time, the target is a cylinder with rectangular cross section of $x, y$ side lengths equal to $d_{r x}=0.15 \lambda$ and $d_{r y}=0.4 \lambda$, respectively, centered at $\left(\rho_{r}, \theta_{r}\right)=(-0.2 \sqrt{2} \lambda, 3 \pi / 4)$. It has a relative dielectric permittivity $\varepsilon_{r, r}=2$ and electric conductivity $\sigma_{r}=10 \mathrm{mS} / \mathrm{m}$. Some examples of the reconstructed dielectric profiles within the cylindrical enclosure and in free space have been shown in Figure 11a,b, respectively. Observing these images, it is confirmed that the PEC enclosure gives the best results even in this configuration, while the reconstruction obtained in free space is worse and more affected by background artifacts. Similar observations can be drawn from the reconstruction errors, reported in Table 2. As it happened with the circular target in the same conditions, both the mean value of the NRE and its standard deviation are significantly lower for the imaging inside cylindrical enclosure.

For comparison purposes, the same data have also been inverted by using an $L^{2}$ Hilbert-space formulation (i.e., with $p=2$ ). Results are presented in Figure 11c,d. Clearly, in both PEC enclosure and free space, the dielectric properties of the target are underestimated. Furthermore, some artifacts appear in Figure 11d. The reconstruction errors listed in Table 2 lead to some interesting remarks. First, in all cases the proposed non-Hilbertian formulation gives by far the best results. Second, benefits of working inside the PEC enclosure still exist in the $L^{2}$ case, but are more evident when the regularization is performed outside Hilbert spaces. Third, the Hilbert-space NREs have a reduced standard deviation compared to the others, which is due to the higher smoothing effect. Although this may mitigate the impact of noise to some extent, it also degrades the reconstruction considerably (over-smoothing).

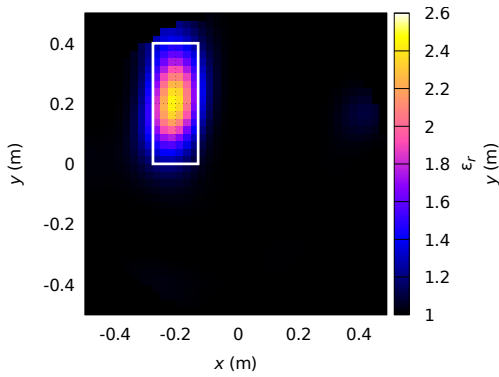

(a)

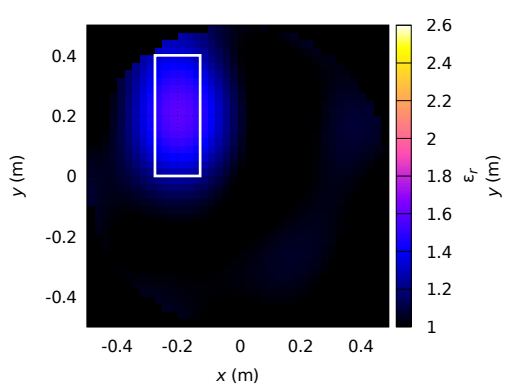

(c)

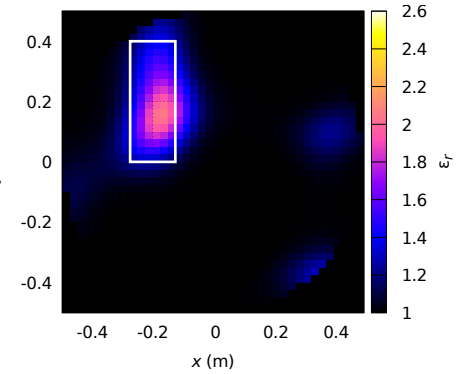

(b)

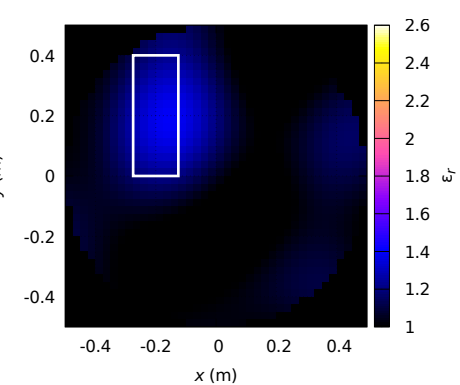

(d)

Figure 11. Rectangular cylinder. Examples of reconstructed distributions of the relative dielectric permittivity with enclosure of radius $a=2.125 \lambda$ and $\sigma_{b}=2 \mathrm{mS} / \mathrm{m}$. Upper row: $L^{p}$-space results, with $p=1.2$. (a) Reconstruction with cylindrical enclosure; (b) Reconstruction in free space. Lower row: Hilbert-space results. (c) Reconstruction with cylindrical enclosure; (d) Reconstruction in free space. 
Table 2 also reports some computational data (time and random access memory) required to run the inversion methods on a personal computer equipped with an Intel(R) Core(TM) i5-2310 CPU at $2.90 \mathrm{GHz}$ (Intel, Santa Clara, CA, USA) and 8 GB of RAM. Computational time is notably higher when a cylindrical enclosure is considered, and it shows a slight (average) increase in the proposed approach due to the number of performed Newton iterations. When the number of iterations is the same, there are no significant differences between methods. Furthermore, the present implementation of the case of PEC enclosure requires more random access memory than the free space case, but the amount of needed RAM is still similar for the $L^{p}$ and the $L^{2}$ methods.

In summary, these results confirm the advantages of adopting a non-Hilbertian formulation for the imaging of targets inside conducting enclosures, and extend the previous analyses carried out in open space configurations [33].

Table 2. Rectangular cylinder. Reconstruction errors (NRE), computational times (mean value \pm standard deviation) and required random access memory (RAM) with the proposed $L^{p}$-space approach compared with a Hilbert-space formulation.

\begin{tabular}{cccc}
\hline & & Proposed Approach & Hilbert-Space \\
\hline \multirow{3}{*}{ Cylindrical enclosure } & NRE & $0.526 \pm 0.020$ & $0.706 \pm 0.008$ \\
& Time (s) & $90.24 \pm 10.60$ & $85.05 \pm 0.203$ \\
& RAM (MB) & $129.4 \pm 0.254$ & $129.5 \pm 0.205$ \\
\hline \multirow{2}{*}{ Free space } & NRE & $0.622 \pm 0.049$ & $0.774 \pm 0.020$ \\
& Time (s) & $12.34 \pm 0.347$ & $8.937 \pm 2.695$ \\
& RAM (MB) & $20.79 \pm 0.233$ & $20.78 \pm 0.240$ \\
\hline
\end{tabular}

\section{Conclusions}

In this paper, the nonlinear microwave imaging of targets enclosed in circular conducting cylinders has been addressed. This particular imaging configuration, which presents some relevant potential applications in industrial nondestructive testing and medical diagnostics, has been considered within an inexact-Newton inversion method formulated in non-Hilbertian $L^{p}$ spaces for the first time. The presence of the circular enclosure has been modeled with a proper Green's function as kernel of the integral operators used to formulate the imaging problem.

A brief discussion about the theoretical background has underlined that the problem faced in this paper is much more tricky than the analogous in free-space. Actually, while in free space the scattering field can be expanded into a simple sum of progressing waves, in the present problem the solution is made by a sum of complicate standing waves, and many resonant modes can arise inside the cavity. Furthermore, also the incident field is strongly affected by the cavity boundaries and contains many modal contributions, depending on the cavity dimensions.

The behavior of the proposed inverse scattering technique when operating in PEC enclosures has been analyzed from a numerical point of view in canonical configurations, involving circular dielectric cylinders. In particular, the simulations have been oriented at understanding the influence of the conducting enclosure on the reconstruction performance. Two distinct classes have been considered, regarding small and large enclosures, with different amounts of background loss.

As expected, for small enclosures without background losses, the first resonances cause a significant degradation of results, impairing a correct reconstruction of the inside dielectric cylinder. On the contrary, in the middle points between these significant resonances, results are comparable or even slightly better (in average) than the free space ones. Instead, when a slightly dissipative background is used, numerical experiments show a completely different behavior, since resonances are smoothed, and the average error seems to show some advantages in using a conducting enclosure with respect to the free space conditions. While, as expected, errors rise when enlarging the enclosure and moving measurement points away from targets, the error increase with the enclosure is slightly less steep than 
the free space case. Moreover, in the lossy case, reconstruction results obtained inside large conducting cylinders are always better then the free space ones, having a smaller mean error on the dielectric characterization of the targets and even a significantly reduced standard deviation. The advantages of embedding the measurement configuration in a metallic cylinder appear more evident if the background losses rise.

As it is well known, the selection of proper operating frequencies represents a crucial problem in time-harmonic microwave imaging. The presence of conducting enclosures further complicates this issue, due to the possible resonance phenomena. If the inversion method does not takes explicitly advantage from resonant modes, and low-loss configurations should be adopted, a general suggestion is to choose the working frequency so as to be sufficiently away from critical resonances, which can be computed based on the behavior of Bessel's functions. However, avoiding resonances may be very difficult as the size of the imaging chamber rises. As proven by the presented results, this problem is mitigated when the infill medium has non-negligible losses.

In conclusion, the adoption of a non-Hilbertian Lebesgue-space formulation seems promising for the imaging inside metallic cylinders, and may lead to more accurate dielectric reconstructions compared to the standard $L^{2}$ approach. Although some relevant differences may be observed based on the size of the outer metallic cylinder, ad-hoc chambers of appropriate dimensions can enhance results in the presence of lossy backgrounds. This could be exploited in many applications where not only boundaries can be controlled, but also the imaging devices can benefit from the use of circular enclosures, such as the case of biomedical imaging where the process requires a tight coupling of the investigated region with the surrounding background media.

Author Contributions: Conceptualization, A.F., M.P., A.R. and G.L.G.; methodology, A.F., M.P., A.R. and G.L.G.; formal analysis, A.F., M.P., A.R. and G.L.G.; investigation, A.F., M.P., A.R. and G.L.G. All authors have read and agreed to the published version of the manuscript.

Funding: This research received no external funding.

Data Availability Statement: The numerical data presented in this study are available from the corresponding author on request.

Conflicts of Interest: The authors declare no conflict of interest.

\section{Abbreviations}

The following abbreviations are used in this manuscript:

PEC Perfect Electric Conductor

TM Transverse Magnetic

NRE Normalized Reconstruction Error

RAM Random Access Memory

\section{References}

1. Kak, A.C.; Slaney, M. Principles of Computerized Tomographic Imaging; IEEE Press: New York, NY, USA, 1988.

2. Benedetto, A.; Pajewski, L. Civil Engineering Applications of Ground Penetrating Radar; Springer: Cham, Switzerland, 2015.

3. Bolomey, J.C. Advancing Microwave-Based Imaging Techniques for Medical Applications in the Wake of the 5G Revolution. In Proceedings of the 13th European Conference on Antennas and Propagation, Krakow, Poland, 31 March-5 April 2019; pp. 1-5.

4. Cakoni, F.; Colton, D. Qualitative Methods in Inverse Scattering Theory: An Introduction; Springer Science \& Business Media: Berlin/Heidelberg, Germany, 2005.

5. Cakoni, F.; Colton, D.L.; Haddar, H. Inverse Scattering Theory and Transmission Eigenvalues; SIAM: Philadelphia, PA, USA, 2016.

6. Nikolova, N.K. Introduction to Microwave Imaging; EuMA High Frequency Technologies Series; Cambridge University Press: Cambridge, UK, 2017. [CrossRef]

7. Pastorino, M.; Randazzo, A. Microwave Imaging Methods and Applications; Artech House: Boston, MA, USA, 2018.

8. Crocco, L.; Litman, A. On embedded microwave imaging systems: Retrievable information and design guidelines. Inverse Probl. 2009, 25, 065001. [CrossRef]

9. Gilmore, C.; LoVetri, J. Enhancement of microwave tomography through the use of electrically conducting enclosures. Inverse Probl. 2008, 24, 035008. [CrossRef] 
10. Coli, V.L.; Tournier, P.H.; Dolean, V.; Kanfoud, I.E.; Pichot, C.; Migliaccio, C.; Blanc-Féraud, L. Detection of simulated brain strokes using microwave tomography. IEEE J. Electromagn. Microw. Med. Biol. 2019, 3, 254-260. [CrossRef]

11. Gilmore, C.; Zakaria, A.; Pistorius, S.; LoVetri, J. Microwave Imaging of Human Forearms: Pilot Study and Image Enhancement. Int. J. Biomed. Imaging 2013, 2013, 673027. [CrossRef] [PubMed]

12. Asefi, M.; Baran, A.; LoVetri, J. An Experimental Phantom Study for Air-Based Quasi-Resonant Microwave Breast Imaging. IEEE Trans. Microw. Theory Tech. 2019, 67, 3946-3954. [CrossRef]

13. Fedeli, A.; Schenone, V.; Randazzo, A.; Pastorino, M.; Henriksson, T.; Semenov, S. Nonlinear S-parameters inversion for stroke imaging. IEEE Trans. Microw. Theory Tech. 2020, in press. [CrossRef]

14. Winges, J.; Cerullo, L.; Rylander, T.; McKelvey, T.; Viberg, M. Compressed Sensing for the Detection and Positioning of Dielectric Objects Inside Metal Enclosures by Means of Microwave Measurements. IEEE Trans. Microw. Theory Tech. 2018, 66, 462-476. [CrossRef]

15. LoVetri, J.; Asefi, M.; Gilmore, C.; Jeffrey, I. Innovations in Electromagnetic Imaging Technology: The Stored-Grain-Monitoring Case. IEEE Antennas Propag. Mag. 2020, 62, 33-42. [CrossRef]

16. Chen, X.; Wei, Z.; Li, M.; Rocca, P. A review of deep learning approaches for inverse scattering problems. Prog. Electromagn. Res. 2020, 167, 67-81. [CrossRef]

17. Pavone, S.C.; Sorbello, G.; Di Donato, L. On the Orbital Angular Momentum Incident Fields in Linearized Microwave Imaging. Sensors 2020, 20, 1905. [CrossRef]

18. Bevacqua, M.T.; Isernia, T.; Palmeri, R.; Akinci, M.N.; Crocco, L. Physical insight unveils new imaging capabilities of orthogonality sampling method. IEEE Trans. Antennas Propag. 2020, 68, 4014-4021. [CrossRef]

19. Donelli, M.; Franceschini, D.; Rocca, P.; Massa, A. Three-dimensional microwave imaging problems solved through an efficient multiscaling particle swarm optimization. IEEE Trans. Geosci. Remote Sens. 2009, 47, 1467-1481. [CrossRef]

20. Fedeli, A.; Maffongelli, M.; Monleone, R.; Pagnamenta, C.; Pastorino, M.; Poretti, S.; Randazzo, A.; Salvadè, A. A tomograph prototype for quantitative microwave imaging: Preliminary experimental results. J. Imaging 2018, 4, 139. [CrossRef]

21. Donelli, M.; Manekiya, M.; Iannacci, J. Development of a MST sensor probe, based on a SP3T switch, for biomedical applications. Microw. Opt. Technol. Lett. 2021, 63, 82-90. [CrossRef]

22. Afsari, A.; Abbosh, A.M.; Rahmat-Samii, Y. Modified Born iterative method in medical electromagnetic tomography using magnetic field fluctuation contrast source operator. IEEE Trans. Microw. Theory Tech. 2019, 67, 454-463. [CrossRef]

23. Mojabi, P.; LoVetri, J. Eigenfunction contrast source inversion for circular metallic enclosures. Inverse Probl. 2010, 26, 025010. [CrossRef]

24. Abdollahi, N.; Jeffrey, I.; LoVetri, J. Non-Iterative Eigenfunction-Based Inversion (NIEI) Algorithm for 2D Helmholtz Equation. Prog. Electromagn. Res. B 2019, 85, 1-25. [CrossRef]

25. Rubek, T.; Meaney, P.M.; Meincke, P.; Paulsen, K.D. Nonlinear microwave imaging for breast-cancer screening using GaussNewton's method and the CGLS inversion algorithm. IEEE Trans. Antennas Propag. 2007, 55, 2320-2331. [CrossRef]

26. Mojabi, P.; LoVetri, J.; Shafai, L. A multiplicative regularized Gauss-Newton inversion for shape and location reconstruction. IEEE Trans. Antennas Propag. 2011, 59, 4790-4802. [CrossRef]

27. Abubakar, A.; Habashy, T.M.; Pan, G.; Li, M.K. Application of the multiplicative regularized Gauss-Newton algorithm for three-dimensional microwave imaging. IEEE Trans. Antennas Propag. 2012, 60, 2431-2441. [CrossRef]

28. Estatico, C.; Fedeli, A.; Pastorino, M.; Randazzo, A. Quantitative microwave imaging method in Lebesgue spaces with nonconstant exponents. IEEE Trans. Antennas Propag. 2018, 66, 7282-7294. [CrossRef]

29. Estatico, C.; Fedeli, A.; Pastorino, M.; Randazzo, A.; Tavanti, E. A phaseless microwave imaging approach based on a Lebesguespace inversion algorithm. IEEE Trans. Antennas Propag. 2020, 68, 8091-8103. [CrossRef]

30. Schöpfer, F.; Louis, A.K.; Schuster, T. Nonlinear iterative methods for linear ill-posed problems in Banach spaces. Inverse Probl. 2006, 22, 311-329. [CrossRef]

31. Estatico, C.; Fedeli, A.; Pastorino, M.; Randazzo, A. Microwave imaging of elliptically shaped dielectric cylinders by means of an Lp Banach-space inversion algorithm. Meas. Sci. Technol. 2013, 24, 074017. [CrossRef]

32. Bisio, I.; Estatico, C.; Fedeli, A.; Lavagetto, F.; Pastorino, M.; Randazzo, A.; Sciarrone, A. Variable-exponent Lebesgue-space inversion for brain stroke microwave imaging. IEEE Trans. Microw. Theory Tech. 2020, 68, 1882-1895. [CrossRef]

33. Estatico, C.; Fedeli, A.; Pastorino, M.; Randazzo, A. Microwave imaging by means of Lebesgue-space inversion: An overview. Electronics 2019, 8, 945. [CrossRef]

34. Van Bladel, J.G. Electromagnetic Fields, 2nd ed.; IEEE Press Series on Electromagnetic Wave Theory; John Wiley \& Sons: Hoboken, NJ, USA, 2007; Volume 19.

35. Balanis, C.A. Advanced Engineering Electromagnetics, 2nd ed.; John Wiley \& Sons: Hoboken, NJ, USA, 2012.

36. Martinek, J.; Thielman, H.P. On Green's functions for the reduced wave equation in a circular annular domain with Dirichlet, Neumann and radiation type boundary conditions. Appl. Sci. Res. 1966, 16, 5-12. [CrossRef]

37. Duffy, D.G. Green's Functions with Applications, 1st ed.; Chapman \& Hall/CRC: Boca Raton, FL, USA, 2001.

38. Kukla, S.; Siedlecka, U.; Zamorska, I. Green's functions for interior and exterior Helmholtz problems. Sci. Res. Inst. Math. Comput. Sci. 2012, 11, 53-62. [CrossRef]

39. Stokes, G.G. On the numerical Calculation of a Class of Definite Integrals and Infinite Series. Trans. Camb. Philos. Soc. 1856, 9, 166-187. 
40. McMahon, J. On the Roots of the Bessel and Certain Related Functions. Ann. Math. 1894, 9, 23-30. [CrossRef]

41. Watson, G.N. The Zeros of Bessel Functions. Proc. R. Soc. London. Ser. A Contain. Pap. Math. Phys. Character 1918, 94, 190-206. [CrossRef]

42. Watson, G.N. A Treatise on the Theory of Bessel Functions, 2nd ed.; Cambridge University Press: Cambridge, UK, $1944 ;$ p. 804.

43. Elbert, Á.; Laforgia, A. An asymptotic relation for the zeros of Bessel functions. J. Math. Anal. Appl. 1984, 98, 502-511. [CrossRef]

44. Ifantis, E.; Siafarikas, P. Inequalities involving Bessel and modified Bessel functions. J. Math. Anal. Appl. 1990, 147, 214-227. [CrossRef]

45. Ifantis, E.; Siafarikas, P. Differential inequalities for the positive zeros of Bessel functions. J. Comput. Appl. Math. 1990, 30, 139-143. [CrossRef]

46. Ifantis, E.; Siafarikas, P. A differential inequality for the positive zeros of Bessel functions. J. Comput. Appl. Math. 1992, 44, 115-120. [CrossRef]

47. Breen, S. Uniform Upper and Lower Bounds on the Zeros of Bessel Functions of the First Kind. J. Math. Anal. Appl. 1995, 196, 1-17. [CrossRef]

48. Elbert, Á. Some recent results on the zeros of Bessel functions and orthogonal polynomials. J. Comput. Appl. Math. 2001, 133, 65-83. [CrossRef]

49. Segura, J. Bounds on Differences of Adjacent Zeros of Bessel Functions and Iterative Relations between Consecutive Zeros. Math. Comput. 2001, 70, 1205-1220. [CrossRef]

50. Pálmai, T.; Apagyi, B. Interlacing of positive real zeros of Bessel functions. J. Math. Anal. Appl. 2011, 375, 320-322. [CrossRef]

51. Kerimov, M.K. Studies on the zeros of Bessel functions and methods for their computation. Comput. Math. Math. Phys. 2014, 54, 1337-1388. [CrossRef]

52. Kokologiannaki, C.G.; Laforgia, A. Simple proofs of classical results on zeros of $\mathbf{J} \bullet(\mathbf{x})$ and J' (x). Tbilisi Math. J. 2014, 7, 35-39. [CrossRef]

53. Kerimov, M.K. Studies on the Zeroes of Bessel Functions and Methods for Their Computation: IV. Inequalities, Estimates, Expansions, etc., for Zeros of Bessel Functions. Comput. Math. Math. Phys. 2018, 58, 1-37. [CrossRef]

54. Joó, I. On the control of a circular membrane. I. Acta Math. Hung. 1993, 61, 303-325. [CrossRef]

55. Liu, H.; Zou, J. Zeros of the Bessel and spherical Bessel functions and their applications for uniqueness in inverse acoustic obstacle scattering. IMA J. Appl. Math. 2007, 72, 817-831. [CrossRef]

56. Kurup, D.G.; Koithyar, A. New Expansions of Bessel Functions of First Kind and Complex Argument. IEEE Trans. Antennas Propag. 2013, 61, 2708-2713. [CrossRef]

57. Beneventano, C.G.; Fialkovsky, I.V.; Santangelo, E.M. Zeros of combinations of Bessel functions and the mean charge of graphene nanodots. Theor. Math. Phys. 2016, 187, 497-510. [CrossRef]

58. Karamehmedović, M.; Kirkeby, A.; Knudsen, K. Stable source reconstruction from a finite number of measurements in the multi-frequency inverse source problem. Inverse Probl. 2018, 34, 065004. [CrossRef]

59. Qu, C.; Wong, R. "Best possible" upper and lower bounds for the zeros of the Bessel function $J_{v}(x)$. Trans. Am. Math. Soc. 1999, 351, 2833-2859. [CrossRef]

60. Ismail, M.E.; Muldoon, M.E. On the variation with respect to a parameter of zeros of Bessel and q-Bessel functions. J. Math. Anal. Appl. 1988, 135, 187-207. [CrossRef]

61. Yousif, H.A.; Melka, R. Bessel function of the first kind with complex argument. Comput. Phys. Commun. 1997, 106, 199-206. [CrossRef]

62. Doring, B. Complex Zeros of Cylinder Functions. Math. Comput. 1966, 20, 215-222. [CrossRef] 